УДК $519.21+62$

\author{
Y. M. Chabanyuk, A. V. Nikitin, U. T. Khimka
}

\title{
ASYMPTOTIC PROPERTIES OF THE IMPULSE PERTURBATION PROCESS WITH CONTROL FUNCTION UNDER LEVY APPROXIMATION CONDITIONS
}

\begin{abstract}
Y. M. Chabanyuk, A. V. Nikitin, U. T. Khimka. Asymptotic properties of the impulse perturbation process under Levy approximation conditions with the point of equilibrium of the quality criterion, Mat. Stud. 52 (2019), 96-104.

For the system of stochastic differential equations with Markov switchings and impulse disturbance in the conditions of Levy approximation of a single equilibrium point of the quality criterion, limiting generators of the impulse process and dynamical system are constructed. In particular, we discuss how the behavior of the boundary process depends on the pre-limiting normalization of the stochastic evolutionary system in the ergodic Markov environment.
\end{abstract}

1. Introduction. We consider some pre-limit evolution models with a small normalization parameter. We find the form of the limit generators for the impulse processes and the dynamical system in the schemes of the Lévy approximation, and the stochastic approximation. Further, we provide conditions which ensure weak convergence of a controlled evolution model with Markov switching and impulse perturbation (assuming uniqueness of the equilibrium point for the quality criterion for which the stochastic approximation procedure is given). It is important that the asymptotic behavior of the limit process is concluded with the help of the analysis of parameters of the pre-limit system.

This work continues the direction of research carried out in papers [4], [6]-[8], in which the asymptotic properties of evolution models were obtained under conditions of non-classical approximation schemes.

2. Formulation of the problem. The stochastic evolution system in the ergodic Markovian environment is given by a stochastic differential equation [7]

$$
d y^{\varepsilon}(t)=C\left(y^{\varepsilon}(t), x\left(t / \varepsilon^{2}\right)\right) d t+d \eta^{\varepsilon}\left(t, u^{\varepsilon}(t)\right),
$$

where $y^{\varepsilon}(t)$ is a random evolution, $x(t), t \geq 0$, is a uniformly ergodic Markovian process, that is defined on a standard phase space $(X, \mathbb{X})$ by the generator

$$
Q \varphi(x)=q(x) \int_{E} P(x, d y)[\varphi(y)-\varphi(x)]
$$

2010 Mathematics Subject Classification: 60H30.

Keywords: random evolution; Levy's approximation conditions; point of equilibrium of the quality criterion. doi:10.30970/ms.52.1.96-104

(C) Y. M. Chabanyuk, A. V. Nikitin, U. T. Khimka, 2019 
on the Banach space $B(X)$ of real valued bounded functions with the supremum norm

$$
\|\varphi(x)\|=\sup _{x \in X}|\varphi(x)|
$$

$u^{\varepsilon}(t)$ is a control function that we define later.

A stochastic kernel $P(x, B), x \in X, B \in \mathbb{X}$ defines uniformly ergodic embedded Markovian chain $x_{n}=x\left(\tau_{n}\right), n \geq 0$ that has stationary distribution $\rho(B), B \in \mathbb{X}$. A stationary distribution $\pi(B), B \in \mathbb{X}$ is defined by relation [3]

$$
\pi(d x) q(x)=q \rho(d x)
$$

where

$$
q=\int_{X} \pi(d x) q(x)
$$

We define $R_{0}$ as a potential operator for generator $Q$ by the relation

$$
R_{0}=\Pi-(\Pi+Q)^{-1}
$$

Here $\Pi \varphi(x)=\int_{X} \pi(d y) \varphi(y) 1(x)$ is a projector on the subspace $N_{Q}=\varphi: Q \varphi=0$ of zeros to $Q$.

3. Impulse perturbation process under Levy's approximation conditions. Under Levy's approximation scheme the impulse perturbation process (IPP) $\eta^{\varepsilon}(t), t \geq 0$, is given by the relation

$$
\eta^{\varepsilon}\left(t, u^{\varepsilon}(t)\right)=\int_{0}^{t} \eta^{\varepsilon}\left(d s, u^{\varepsilon}(s), x\left(t / \varepsilon^{2}\right)\right)
$$

where a set of processes with independent increments $\eta^{\varepsilon}(t, u, x), t \geq 0, x \in X, u \in \mathbb{R}$ is determined by the generators

$$
\Gamma^{\varepsilon}(x) \varphi(u, w)=\varepsilon^{-2} \int_{\mathbb{R}}(\varphi(u, w+v)-\varphi(u, w)) \Gamma^{\varepsilon}(d v, x), x \in X
$$

and the following Levy's approximation conditions are satisfied $([2,3])$ :

L1. Approximation of averages

$$
\int_{\mathbb{R}} v \Gamma^{\varepsilon}(d v, x)=\varepsilon a_{1}(x)+\varepsilon^{2}\left(a_{2}(x)+\theta_{a}(x)\right), \theta_{a}(x) \rightarrow 0, \varepsilon \rightarrow 0,
$$

and

$$
\int_{\mathbb{R}} v^{2} \Gamma^{\varepsilon}(d v, x)=\varepsilon\left(b(x)+\theta_{b}(x)\right), \theta_{b}(x) \rightarrow 0, \varepsilon \rightarrow 0,
$$

L2. Condition on the distribution function

$$
\int_{\mathbb{R}} g(v) \Gamma^{\varepsilon}(d v, x)=\varepsilon^{2}\left(\Gamma_{g}(x)+\theta_{g}(x)\right), \theta_{g}(x) \rightarrow 0, \varepsilon \rightarrow 0,
$$


for each $g(v) \in C^{2}(\mathbb{R})$ (space of bounded functions with values in $\mathbb{R}$ and $g(v) /|v|^{2} \rightarrow 0$, $|v| \rightarrow 0)$. Measure $\Gamma_{g}(x)$ is bounded for each $g(v) \in C^{2}(\mathbb{R})$ and can be determined by relation

$$
\Gamma_{g}(x)=\int_{\mathbb{R}} g(v) \Gamma_{0}(d v, x), g(v) \in C^{2}(\mathbb{R}) ;
$$

L3. Uniform quadratic integrability

$$
\sup \lim _{c \rightarrow \infty} \int_{|v|>c} v^{2} \Gamma_{0}(d v, x)=0
$$

4. Stochastic approximation procedure. Let the criterion of the process quality be determined by the function $G(y, x, u)$ that has a unique point of equilibrium $u_{x}^{*}$ on interval $\left[\tau_{i}, \tau_{i+1}\right]$. It follows from condition

$$
G(y(t), x(t), u(t))=0 \text {. }
$$

We note that the solution of stochastic equation (1) on $\left[\tau_{i}, \tau_{i+1}\right]$ forms the Markov process with non-random control.

For the problem (1), (4) we consider the stochastic approximation procedure

$$
d u^{\varepsilon}(t)=\alpha(t) G\left(y^{\varepsilon}(t), x\left(t / \varepsilon^{2}\right), u^{\varepsilon}(t)\right) d t .
$$

where we assume that the function $\alpha(t)>0$ satisfies the following conditions

$\mathrm{P} 1: \quad \int_{t_{0}}^{\infty} \alpha(t) d t=\infty, t_{0}>0$

P2: $\quad \int_{t_{0}}^{\infty} \alpha^{2}(t) d t<\infty, t_{0}>0$.

Now we can formulate the main result of our paper.

Theorem 1. Let the balance condition

$$
\hat{a}_{1}:=\int_{X} \pi(d x) a_{1}(x)=0
$$

and conditions L1-L3 hold. Then the weak convergence

$$
\left(y^{\varepsilon}(t), u^{\varepsilon}(t), \eta^{\varepsilon}(t)\right) \Rightarrow(\hat{y}(t), \hat{u}(t), \hat{\eta}(t)), \varepsilon \rightarrow 0 .
$$

is true. The limit process $(\hat{y}(t), \hat{u}(t), \hat{\eta}(t))$ is determined by generator

$$
\mathbf{M} \varphi(y, u, w)=\mathbf{L} \varphi(y, u, w)+\mathbf{B} \varphi(y, u, w)
$$

where

$$
\begin{gathered}
\mathbf{L} \varphi(y, u, w)=C(y) \varphi(y, u, w)+\Gamma \varphi(y, u, w), \\
C(y) \varphi(y)=\hat{C}(y) \varphi^{\prime}(y), \hat{C}(y)=\int_{X} \pi(d x) C(y, x),
\end{gathered}
$$




$$
\begin{gathered}
\Gamma \varphi(u, w)=\hat{a}_{2} \varphi_{w}^{\prime}(u, w)+\frac{1}{2} \sigma^{2} \varphi_{w w}^{\prime \prime}(u, w)+\int_{\mathbb{R}}[\varphi(u, w+v)-\varphi(u, w)] \hat{\Gamma}_{0}(d v), \\
\hat{a}_{2}=\int_{X} \pi(d x)\left(a_{2}(x)-a_{0}(x)\right), \sigma^{2}=\int_{X} \pi(d x)\left(b(x)-b_{0}(x)\right)+2 \int_{X} \pi(d x) a_{1}(x) R_{0} a_{1}(x), \\
a_{0}(x)=\int_{\mathbb{R}} v \Gamma_{0}(d v, x), b_{0}(x)=\int_{\mathbb{R}} v^{2} \Gamma_{0}(d v, x), \hat{\Gamma}_{0}(v)=\int_{X} \Gamma_{0}(d v, x) ; \\
\mathbf{B} \varphi(y, u, w)=\alpha(t) G(y, u) \varphi_{u}^{\prime}(y, u, w) ; \quad G(y, u)=\int_{X} G(y, x, u) \pi(d x) .
\end{gathered}
$$

Proof of Theorem 1. Firstly we investigate some properties of generators with independent increments.

Lemma 1. Under conditions $\mathbf{L} 1$ - L3 generators with independent increments $\eta^{\varepsilon}(t, u, x)$, $t \geq 0, x \in X, u \in \mathbb{R}$ can be represented as follows

$$
\Gamma^{\varepsilon}(x) \varphi(u, w)=\varepsilon^{-1} \Gamma_{1}(x) \varphi(u, w)+\Gamma_{2}(x) \varphi(u, w)
$$

on test functions $\varphi(u, w) \in \mathbb{R} \times C_{3}(\mathbb{R})$. Here

$$
\begin{gathered}
\Gamma_{1}(x) \varphi(u, w)=a_{1}(x) \varphi_{w}^{\prime}(u, w) \\
\Gamma_{2}(x) \varphi(u, w)=\left(a_{2}(x)-a_{0}(x)\right) \varphi_{w}^{\prime}(u, w)+\frac{1}{2}\left(b(x)-b_{0}(x)\right) \varphi_{w w}^{\prime \prime}(u, w)+ \\
+\int_{\mathbb{R}}[\varphi(u, w+v)-\varphi(u, w)] \Gamma_{0}(d v, x) .
\end{gathered}
$$

Proof. We use the Taylor series of the function $\varphi(u, w)$ and transform generator (3)

$$
\begin{gathered}
\Gamma^{\varepsilon}(x) \varphi(u, w)=\varepsilon^{-2} \int_{\mathbb{R}}(\varphi(u, w+v)-\varphi(u, w)) \Gamma^{\varepsilon}(d v, x)= \\
=\varepsilon^{-2} \int_{\mathbb{R}}\left(\varphi(u, w+v)-\varphi(u, w)-v \varphi_{w}^{\prime}(u, w)-\frac{1}{2} v^{2} \varphi_{w w}^{\prime \prime}(u, w)\right) \Gamma^{\varepsilon}(d v, x)+ \\
\left.+\varepsilon^{-2} \int_{\mathbb{R}} v \varphi_{w}^{\prime}(u, w) \Gamma^{\varepsilon}(d v, x)+\frac{\varepsilon^{-2}}{2} \int_{\mathbb{R}} v^{2} \varphi_{w w}^{\prime \prime}(u, w)\right) \Gamma^{\varepsilon}(d v, x)= \\
=\int_{\mathbb{R}}\left(\varphi(u, w+v)-\varphi(u, w)-v \varphi_{w}^{\prime}(u, w)-\frac{1}{2} v^{2} \varphi_{w w}^{\prime \prime}(u, w)\right) \Gamma^{0}(d v, x)+ \\
+\varepsilon^{-1} a_{1}(x) \varphi_{w}^{\prime}(u, w)+a_{2}(x) \varphi_{w}^{\prime}(u, w)+\frac{1}{2} b(x) \varphi_{w w}^{\prime \prime}(u, w)+\gamma^{\varepsilon}(x) \varphi(u, w)= \\
=\varepsilon^{-1} a_{1}(x) \varphi_{w}^{\prime}(u, w)+\left(a_{2}(x)-a_{0}(x)\right) \varphi_{w}^{\prime}(u, w)+\frac{1}{2}\left(b(x)-b_{0}(x)\right) \varphi_{w w}^{\prime \prime}(u, w)+ \\
+\int_{\mathbb{R}}(\varphi(u, w+v)-\varphi(u, w)) \Gamma^{0}(d v, x)+\gamma^{\varepsilon}(x) \varphi(u, w),
\end{gathered}
$$

where next to the last equality follows from Levy's conditions L1-L3. 
We notice that the function $\varphi(\cdot, w+v)-\varphi(\cdot, w)-v \varphi_{w}^{\prime}(\cdot, w)-\frac{1}{2} v^{2} \varphi_{w w}^{\prime \prime}(\cdot, w+v) \in C_{3}(\mathbb{R})$ because it is bounded and $\varphi(\cdot, w)$ is bounded with its derivatives in variable $w$ and

$$
\lim _{|v| \rightarrow 0} \frac{\left[\varphi(u, w+v)-\varphi(u, w)-v \varphi_{w}^{\prime}(u, w)-\frac{1}{2} v^{2} \varphi_{w w}^{\prime \prime}(u, w+v)\right]}{|v|^{2}}=0 .
$$

Reminding $\gamma^{\varepsilon}(u, w) \varphi(u, w)=O\left(\varepsilon^{2}\right)$, we obtain (5).

Lemma 2. Generator of three component Markov process $\left(u^{\varepsilon}, \eta^{\varepsilon}, x\right), t \geq 0$ has a form

$$
\begin{gathered}
\hat{\Gamma}^{\varepsilon}(x) \varphi(u, w, x)=\varepsilon^{-2} Q \varphi(u, w, x)+\varepsilon^{-1} \Gamma_{1}(x) \varphi(u, w, x)+\Gamma_{1}(x) \varphi(u, w, x)+ \\
+\gamma^{\varepsilon}(x) \varphi(u, w, x),
\end{gathered}
$$

where operators $\Gamma_{1}(x), \Gamma_{2}(x)$ are determined in Lemma 1, and the reminder term

$$
\left\|\gamma^{\varepsilon}(x) \varphi(u, w, x)\right\| \rightarrow 0
$$

as $\varepsilon \rightarrow 0, \varphi(\cdot, w, \cdot) \in \mathbb{C}(\mathbb{R})$.

Proof. Lemma 2 becomes clear using definitions of generator of Markov process and forms of generators of $\eta^{\varepsilon}, u^{\varepsilon}$ and $x$.

We introduce a truncated generator [8]

$$
\Gamma_{0}^{\varepsilon}(x) \varphi(u, w, x)=\varepsilon^{-2} Q \varphi(u, w, x)+\varepsilon^{-1} \Gamma_{1}(x) \varphi(u, w, x)+\Gamma_{1}(x) \varphi(u, w, x) .
$$

Lemma 3. Under balance condition (4), the solution of the singular perturbation problem for truncated operator is realized by the relation

$$
\Gamma_{0}^{\varepsilon}(x) \varphi^{\varepsilon}(u, w, x)=\Gamma \varphi(u, w)+\varepsilon \theta_{\eta}^{\varepsilon}(x) \varphi(u, w)
$$

on test functions

$$
\varphi^{\varepsilon}(u, w, x)=\varphi(u, w)+\varepsilon \varphi_{1}(u, w, x)+\varepsilon^{2} \varphi_{2}(u, w, x),
$$

and the reminder term $\theta_{\eta}^{\varepsilon}(x) \varphi(u, w, x)$ is uniformly bounded at $x$.

The limited operator is determined by formula

$$
\Gamma=\Pi \Gamma_{1}(x) R_{0} \Gamma_{1}(x) \Pi+\Pi \Gamma_{2}(x) \Pi .
$$

Proof. For validity equality (11), it is necessary that the coefficients with the same degrees of $\varepsilon$ from left-hand side and right-hand side become equal. Let us calculate

$$
\begin{gathered}
\Gamma_{0}^{\varepsilon}(x) \varphi^{\varepsilon}(u, w, x)=\varepsilon^{-2} \mathbf{Q} \varphi(u, w)+\varepsilon^{-1}\left[\mathbf{Q} \varphi_{1}(u, w, x)+\Gamma_{1}(x) \varphi(u, w)\right]+ \\
+\left[\mathbf{Q} \varphi_{2}(u, w, x)+\Gamma_{1}(x) \varphi_{1}(u, w, x)+\Gamma_{2}(x) \varphi(u, w)\right]+ \\
+\varepsilon\left[\Gamma_{1}(x) \varphi_{2}(u, w, x)+\Gamma_{2}(x) \varphi_{1}(u, w, x)\right]+\varepsilon^{2} \Gamma_{2}(x) \varphi_{2}(u, w, x) .
\end{gathered}
$$

From the first summand follows relation

$$
\mathbf{Q} \varphi(u, w)=0 \Leftrightarrow \varphi(u, w) \in N_{Q} .
$$

and we can see that $\varphi(u, w)$ does not depend on $x$. 
The balance condition is a condition of solvability of the equation

$$
\mathbf{Q} \varphi_{1}(u, w, x)+\Gamma_{1}(x) \varphi(u, w)=0 .
$$

Then

$$
\varphi_{1}(u, w, x)=R_{0} \Gamma_{1}(x) \varphi(u, w) .
$$

Let us consider equation

$$
\mathbf{Q} \varphi_{2}(u, w, x)+\Gamma_{1}(x) \varphi_{1}(u, w, x)+\Gamma_{2}(x) \varphi(u, w)=\Gamma \varphi(u, w)
$$

It can be rewritten in the form

$$
\mathbf{Q} \varphi_{2}(u, w, x)+\Gamma_{1}(x) R_{0} \Gamma_{1}(x) \varphi(u, w)+\Gamma_{2}(x) \varphi(u, w)=\Gamma \varphi(u, w) .
$$

From the solvability condition in the last equation we can find the limited operator in form (12). Then

$$
\varphi_{2}(u, w, x)=R_{0}\left[\Gamma_{1}(x) R_{0} \Gamma_{1}(x)+\Gamma_{2}(x)-\Gamma\right] \varphi(u, w) .
$$

Using (13) and (14), another terms of Tailor series can be written as follows

$$
\begin{gathered}
\varepsilon\left[\Gamma_{1}(x) \varphi_{2}(u, w, x)+\Gamma_{2}(x) \varphi_{1}(u, w, x)\right]+\varepsilon^{2} \Gamma_{2}(x) \varphi_{2}(u, w, x)= \\
\quad=\varepsilon\left[\Gamma_{1}(x) R_{0}\left[\Gamma_{1}(x) R_{0} \Gamma_{1}(x)+\Gamma_{2}(x)-\Gamma\right]+\Gamma_{2}(x) R_{0} \Gamma_{1}(x)\right]+ \\
+\varepsilon \Gamma_{2}(x) R_{0}\left[\Gamma_{1}(x) R_{0} \Gamma_{1}(x)+\Gamma_{2}(x)-\Gamma\right] \varphi(u, w)=\varepsilon \theta_{\eta}^{\varepsilon}(x) \varphi(u, w) .
\end{gathered}
$$

The boundedness of $\theta_{\eta}^{\varepsilon}(x) \varphi(u, w)$ follows from representation of operators $\Gamma_{1}, \Gamma_{2}$ and $R_{0}$.

Lemma 4. A generator of four-component Markov process $\left(y^{\varepsilon}, u^{\varepsilon}, \eta^{\varepsilon}, x\right), t \geq 0$ has the form

$$
\begin{aligned}
& \mathbf{L}^{\varepsilon}(x) \varphi(y, u, w, x)=\varepsilon^{-2} Q \varphi(y, u, w, x)+\Gamma^{\varepsilon}(x) \varphi(y, u, w, x)+ \\
& +\mathbf{C}(x) \varphi(y, u, w, x)+\mathbf{B}(x) \varphi(y, u, w, x)+\theta_{w}^{\varepsilon}(x) \varphi(y, u, w, x),
\end{aligned}
$$

where $\Gamma^{\varepsilon}(x)$ is a generator of set impulse perturbation processes (3),

$$
\mathbf{C}(x) \varphi(y, x)=C(y, x) \varphi_{y}^{\prime}(y, x), \mathbf{B}(x) \varphi(u)=\alpha(t) G(y, x, u) \varphi^{\prime}(u) .
$$

The reminder term $\left\|\theta_{w}^{\varepsilon}(x) \varphi(u, w, x)\right\| \rightarrow 0$ as $\varepsilon \rightarrow 0$.

The proof of Lemma 4 can be found in [7].

Lemma 5. A generator $\mathbf{L}^{\varepsilon}(x)$ in the case of impulse perturbation process has an asymptotic representation

$$
\begin{gathered}
\mathbf{L}^{\varepsilon}(x) \varphi(u, w, x)=\varepsilon^{-2} \mathbf{Q} \varphi(u, w, x)+\varepsilon^{-1} \Gamma_{1}(x) \varphi(u, w, x)+ \\
+\Gamma_{2}(x) \varphi(u, w, x)+\mathbf{C}(x) \varphi(u, w, x)+\mathbf{B}(x) \varphi(u, w, x)+\hat{\theta}_{w}^{\varepsilon} \varphi(u, w, x)
\end{gathered}
$$

where

$$
\hat{\theta}_{w}^{\varepsilon}(x)=\gamma^{\varepsilon}+\theta_{w}^{\varepsilon}(x),
$$

and $\Gamma_{1}(x), \Gamma_{2}(x)$ are determined in Lemma 1.

The reminder term $\left\|\hat{\theta}_{w}^{\varepsilon}(x) \varphi(u, w, x)\right\| \rightarrow 0$ as $\varepsilon \rightarrow 0$. 
The proof of Lemma 5 is carried out using representation of operator (5) and statements of Lemma 4.

A truncated generator has the form

$$
\begin{aligned}
\mathbf{L}_{0}^{\varepsilon}(x) \varphi(u, w, x)= & \varepsilon^{-2} Q \varphi(u, w, x)+\varepsilon^{-1} \Gamma_{1}(x) \varphi(u, w, x)+\Gamma_{2}(x) \varphi(u, w, x)+ \\
& +\mathbf{C}(x) \varphi(u, w, x)+\mathbf{B}(x) \varphi(u, w, x) .
\end{aligned}
$$

Lemma 6. Under balance condition (4), the solution of singular perturbation problem for truncated operator (18) is realized by relation

$$
\mathbf{L}_{0}^{\varepsilon}(x) \varphi^{\varepsilon}(y, u, w, x)=\mathbf{L} \varphi(y, u, w)+\varepsilon^{2} \theta_{w}^{\varepsilon}(x) \varphi(y, u, w)
$$

on test functions

$$
\varphi^{\varepsilon}(y, u, w, x)=\varphi(y, u, w)+\varepsilon \varphi_{1}(y, u, w, x)+\varepsilon^{2} \varphi_{2}(y, u, w, x),
$$

and the reminder term $\theta_{w}^{\varepsilon}(x)$ is uniformly bounded at $x$.

The limited operator is determined by formula

$$
\mathbf{L}=\Pi\left[\mathbf{C}(x)+\Gamma_{1}(x) R_{0} \Gamma_{1}(x)+\Gamma_{2}(x)+\mathbf{B}(x)\right] \Pi .
$$

Proof. For validity of equality (17), it is necessary that the coefficients of the same degrees of $\varepsilon$ from left-hand side and right-hand side become same. We calculate:

$$
\begin{gathered}
\mathbf{L}_{0}^{\varepsilon}(x) \varphi^{\varepsilon}(y, u, w, x)=\varepsilon^{-2} \mathbf{Q} \varphi(y, u, w)+\varepsilon^{-1}\left[\mathbf{Q} \varphi_{1}(y, u, w, x)+\Gamma_{1}(x) \varphi(y, u, w)\right]+ \\
+\left[\mathbf{Q} \varphi_{2}(y, u, w, x)+\Gamma_{1}(x) \varphi_{1}(y, u, w, x)+\right. \\
\left.+\Gamma_{2}(x) \varphi(y, u, w)+\mathbf{C}(x) \varphi(y, u, w)+\mathbf{B} \varphi(y, u, w)\right]+ \\
+\varepsilon\left[\Gamma_{1}(x) \varphi_{2}(y, u, w, x)+\Gamma_{2}(x) \varphi_{1}(y, u, w, x)+\mathbf{C}(x) \varphi_{1}(y, u, w, x)\right]+ \\
+\varepsilon^{2}\left[\Gamma_{2}(x) \varphi_{2}(y, u, w, x)+\mathbf{C}(x) \varphi_{2}(y, u, w, x)+\mathbf{B} \varphi_{2}(y, u, w, x)\right] .
\end{gathered}
$$

Since

$$
\mathbf{Q} \varphi(y, u, w)=0 \Leftrightarrow \varphi(y, u, w) \in N_{Q},
$$

$\varphi(y, u, w)$ does not depend on $x$.

The balance condition is a condition of solvability of equation

$$
\mathbf{Q} \varphi_{1}(y, u, w, x)+\Gamma_{1}(x) \varphi(y, u, w)=0 .
$$

Then $\varphi_{1}(y, u, w, x)=R_{0} \Gamma_{1}(x) \varphi(y, u, w)$.

We rewrite equation

$$
\begin{gathered}
\mathbf{Q} \varphi_{2}(y, u, w, x)+\Gamma_{1}(x) \varphi_{1}(y, u, w, x)+ \\
+\Gamma_{2}(x) \varphi(y, u, w)+\mathbf{C}(x) \varphi(y, u, w)+\mathbf{B}(x) \varphi(y, u, w)=\mathbf{L} \varphi(y, u, w)
\end{gathered}
$$

in the form

$$
\mathbf{Q} \varphi_{2}(y, u, w, x)=\left[\mathbf{L}-\Gamma_{1}(x) R_{0} \Gamma_{1}(x)-\Gamma_{2}(x)-\mathbf{C}(x)-\mathbf{B}(x)\right] \varphi(y, u, w) .
$$

From the solvability condition in the last equation we can find limited operator of the form (20). 
In view of Theorem 6.3 from [3], the proof of Theorem 1 is completed.

Remark 1. Weak convergence of process $\left(y^{\varepsilon}(t), u^{\varepsilon}(t), \eta^{\varepsilon}(t)\right) \Rightarrow(\hat{y}(t), \hat{u}(t), \hat{\eta}(t)), \varepsilon \rightarrow 0$ follows from convergence of the corresponding generators under condition of compactness of pre-limited set of processes $\left(y^{\varepsilon}(t), u^{\varepsilon}(t)\right)$. In particular, the corresponding theorems on the compactness of processes with independent increments in Levy approximation scheme have been proved in [4].

Theorem 2. We assume that conditions L1-L2, P1, P2 are true and there exists Lyapunov function $V(y, u)$ of averaging system

$$
\frac{d u}{d t}=G(y, u)
$$

satisfying the conditions of exponential stability

$$
\text { Y1: } G(y, u) V^{\prime}(y, u) \leq-c V(y, u),
$$

additional conditions for the Lyapunov function

$$
Y 2:\left|C(y, x) R_{0}\left[\tilde{G}(y, x, u) V_{u}^{\prime}(y, u)\right]_{u}^{\prime}\right| \leq c_{1}(1+V(y, u)),
$$

and the following conditions

$$
\begin{gathered}
\left|G(y, x, u) R_{0}\left[\tilde{C}(y, x) V_{u}^{\prime}(y, u)\right]_{u}^{\prime}\right| \leq c_{2}(1+V(y, u)) \\
\left|\tilde{C}(y, x) R_{0}\left[\tilde{C}(y, x) V_{u}^{\prime \prime}(y, u)\right]_{u}^{\prime}\right| \leq c_{3}(1+V(y, u)) \\
\left|G(y, x, u) R_{0}\left[\tilde{G}(y, x, u) V_{u}^{\prime}(y, u)\right]_{u}^{\prime}\right| \leq c_{4}(1+V(y, u)) .
\end{gathered}
$$

Then $\forall \varepsilon>0, \varepsilon \leq \varepsilon_{0}, \varepsilon_{0}>0$ the relation

$$
P\left\{\lim _{t \rightarrow \infty} u(t)=u^{*}\right\}=1, \forall y
$$

is valid.

Proof. For the perturbed Lyapunov function

$$
V^{\varepsilon}(y, u, w, x)=V(y, u)+\varepsilon V_{1}(y, u, w, x)+\varepsilon^{2} V_{2}(y, u, w, x),
$$

using (20), we obtain limiting presentation for control

$$
L_{u}^{\varepsilon} V^{\varepsilon}(y, u, w, x)=\mathbf{B} V(y, u)+\varepsilon \theta_{u}(x) .
$$

From the conditions $Y 1, Y 2$ we have an estimate

$$
L_{u}^{\prime} V^{\varepsilon}(y, u, w, x) \leq-c \alpha(t) V(y, u)+c^{*} \alpha^{2}(t)(1+V(y, u))
$$

and then we use the result of Nevelson-Chasminsky theorem [10]. The proof of Theorem 2 is complete.

Remark 2. The stochastic approximation procedure for control $u$ gives optimal value $u_{y}$ for any IPP $y$. 


\section{REFERENCES}

1. Jacod J., Shiryaev A.N., Limit theorems for stochastic processes, Springer-Verlag, Berlin, 2003 , 601 p.

2. Korolyuk V.S., Korolyuk V.V., Stochastic models of systems, Kluwer, Dordrecht, 1999, 185 p.

3. Korolyuk V.S., Limnios N., Stochastic systems in merging phase space, World Scientific, 2005, 330 p.

4. Korolyuk V.S., Limnios N., Samoilenko I.V., Levy and Poisson approximations of switched stochastic systems by a semimartingale approach, Comptes Rendus Mathematique, 354 (2016), 723-728.

5. Papanicolaou G., Stroock D., Varadhan S.R.S., Martingale approach to some limit theorems, Duke turbulence conference (Durham, NC, April 23-25, 1976), Duke University Mathematics Series III, New York: Duke University, 1977, 120 p.

6. Samoilenko A.M., Stanzhytskyi O.M., Qualitative and asymptotic analysis of differential equations with random perturbations, World Scientific, Singapore, 2011, 323 p.

7. Samoilenko I.V., Chabanyuk Y.M., Nikitin A.V., Khimka U.T., Samoilenko A.M., Differential equations with small stochastic additions under Poisson approximation conditions, Cybernetics and System analysis, 53 (2017), №3, 410-416.

8. Samoilenko I.V., Nikitin A.V., Differential equations with small stochastic terms under the levy approximating conditions, Ukrainian Mathematical Journal, 69 (2018), №9, 1445-1454.

9. Nikitin A.V., Khimka U.T., Asymptotics of normalized control with Markov switchings, Ukrainian Mathematical Journal, 68 (2017), №8, 1252-1262.

10. Nevelson M.B., Khasminskii R.Z., Stochastic approximation and recurrent estimation, M.: Nauka, 1972.

Politechnika Lubelska, Lublin, Poland

yaroslav.chab@gmail.com

Taras Shevchenko National University of Kyiv, Kyiv, Ukraine

nikitin2505@gmail.com

Ivan Franko National University of Lviv, Lviv, Ukraine

ulyana.himka@gmail.com 